\title{
Therapeutic Area
}

National Cancer Institute

\section{Source}

National Cancer Institute. Therapeutic Area. NCI Thesaurus. Code C101302.

A knowledge field that focuses on research and development of treatments for diseases and pathologic findings, as well as prevention of conditions that negatively impact the health of an individual. 